\title{
New results and applications on the existence results for nonlinear coupled systems
}

\author{
Imran Talib1, Thabet Abdeljawad $2,3,4^{*}$ (D, Manar A. Alqudah ${ }^{5}$, Cemil Tunc ${ }^{6}$ and Rabia Ameen ${ }^{1}$
}

\author{
"Correspondence: \\ tabdeljawad@psu.edu.sa \\ ${ }^{2}$ Department of Mathematics and \\ General Sciences, Prince Sultan \\ University, Riyadh, Saudi Arabia \\ ${ }^{3}$ Department of Medical Research, \\ China Medical University, 40402, \\ Taichung, Taiwan \\ Full list of author information is \\ available at the end of the article
}

\begin{abstract}
In this manuscript, we study a certain classical second-order fully nonlinear coupled system with generalized nonlinear coupled boundary conditions satisfying the monotone assumptions. Our new results unify the existence criteria of certain linear and nonlinear boundary value problems (BVPs) that have been previously studied on a case-by-case basis; for example, Dirichlet and Neumann are special cases. The common feature is that the solution of each BVPs lies in a sector defined by well-ordered coupled lower and upper solutions. The tools we use are the coupled lower and upper solutions approach along with some results of fixed point theory. By means of the coupled lower and upper solutions approach, the considered BVPs are logically modified to new problems, known as modified BVPs. The solution of the modified BVPs leads to the solution of the original BVPs. In our case, we only require the Nagumo condition to get a priori bound on the derivatives of the solution function. Further, we extend the results presented in (Franco et al. in Extr. Math. 18(2):153-160, 2003; Franco et al. in Appl. Math. Comput. 153:793-802, 2004; Franco and O'Regan in Arch. Inequal. Appl. 1:423-430, 2003; Asif et al. in Bound. Value Probl. 2015:134, 2015). Finally, as an application, we consider the fully nonlinear coupled mass-spring model.
\end{abstract}

MSC: Primary 34B15; secondary 46B25; 34A30

Keywords: Fully nonlinear coupled mass-spring model; Generalized nonlinear coupled boundary conditions; Lower and upper solutions approach; Dirichlet boundary conditions; Neumann boundary conditions

\section{Springer}

\section{Introduction}

We establish the existence criteria to investigate the existence of solution for the fully nonlinear second-order coupled systems of the type

$$
\begin{array}{ll}
-v_{1}^{\prime \prime}(s)=h_{1}\left(s, v_{1}(s), v_{2}(s), v_{1}^{\prime}(s), v_{2}^{\prime}(s)\right), & s \in[0,1], \\
-v_{2}^{\prime \prime}(s)=h_{2}\left(s, v_{1}(s), v_{2}(s), v_{1}^{\prime}(s), v_{2}^{\prime}(s)\right), & s \in[0,1],
\end{array}
$$

(c) The Author(s) 2021. This article is licensed under a Creative Commons Attribution 4.0 International License, which permits use, sharing, adaptation, distribution and reproduction in any medium or format, as long as you give appropriate credit to the original author(s) and the source, provide a link to the Creative Commons licence, and indicate if changes were made. The images or other third party material in this article are included in the article's Creative Commons licence, unless indicated otherwise in a credit line to the material. If material is not included in the article's Creative Commons licence and your intended use is not permitted by statutory regulation or exceeds the permitted use, you will need to obtain permission directly from the copyright holder. To view a copy of this licence, visit http://creativecommons.org/licenses/by/4.0/. 
subject to the generalized nonlinear boundary conditions (NBCs) of the type

$$
\begin{aligned}
& \Upsilon_{1}\left(v_{1}(0), v_{2}(0), v_{1}(1), v_{2}(1), v_{1}^{\prime}(0), v_{2}^{\prime}(0)\right)=(0,0), \\
& \Upsilon_{2}\left(v_{1}(0), v_{2}(0), v_{1}(1), v_{2}(1), v_{1}^{\prime}(1), v_{2}^{\prime}(1)\right)=(0,0),
\end{aligned}
$$

where $h_{1}, h_{2}:[0,1] \times \mathbb{R}^{4} \rightarrow \mathbb{R}$, and $\Upsilon_{1}, \Upsilon_{2}: \mathbb{R}^{6} \rightarrow \mathbb{R}^{2}$ are continuous functions.

The nonlinear boundary value problems (NBVPs), (1)-(2) include some special cases, for instance, if $\Upsilon_{1}\left(a_{1}, a_{2}, a_{3}, a_{4}, a_{5}, a_{6}\right)=\left(\alpha_{1}-a_{1}, \alpha_{2}-a_{2}\right)$ and $\Upsilon_{2}\left(a_{1}, a_{2}, a_{3}, a_{4}, a_{5}, a_{6}\right)=\left(\alpha_{3}-\right.$ $a_{3}, \alpha_{4}-a_{4}$ ) with $\alpha_{1}, \alpha_{2}, \alpha_{3}, \alpha_{4} \in \mathbb{R}$, then (1)-(2) is the Dirichlet boundary value problem (BVP) with

$$
\begin{array}{ll}
v_{1}(0)=\alpha_{1}, & v_{2}(0)=\alpha_{2}, \\
v_{1}(1)=\alpha_{3}, & v_{2}(1)=\alpha_{4} .
\end{array}
$$

If $\Upsilon_{1}\left(a_{1}, a_{2}, a_{3}, a_{4}, a_{5}, a_{6}\right)=\left(-\alpha_{1}+a_{5},-\alpha_{2}+a_{6}\right)$ and $\Upsilon_{2}\left(a_{1}, a_{2}, a_{3}, a_{4}, a_{5}, a_{6}\right)=\left(-\alpha_{3}+\right.$ $\left.a_{5},-\alpha_{4}+a_{6}\right)$ with $\alpha_{1}, \alpha_{2}, \alpha_{3}, \alpha_{4} \in \mathbb{R}$, then (1)-(2) is the Neumann BVP with

$$
\begin{array}{ll}
v_{1}^{\prime}(0)=\alpha_{1}, & v_{2}^{\prime}(0)=\alpha_{2}, \\
v_{1}^{\prime}(1)=\alpha_{3}, & v_{2}^{\prime}(1)=\alpha_{4} .
\end{array}
$$

If $\Upsilon_{1}\left(a_{1}, a_{2}, a_{3}, a_{4}, a_{5}, a_{6}\right)=\left(\alpha_{1} a_{1}-\alpha_{2} a_{5}-b_{1}, \alpha_{1} a_{2}-\alpha_{2} a_{6}-b_{2}\right)$ and $\Upsilon_{2}\left(a_{1}, a_{2}, a_{3}, a_{4}, a_{5}, a_{6}\right)=$ $\left(\alpha_{3} a_{3}+\alpha_{4} a_{5}-b_{1}, \alpha_{3} a_{4}+\alpha_{4} a_{6}+b_{2}\right)$ with $\alpha_{1}, \alpha_{2}, \alpha_{3}, \alpha_{4}, b_{1}, b_{2} \in \mathbb{R}$, then (1)-(2) is the mixed BVP with

$$
\begin{array}{ll}
\alpha_{1} v_{1}(0)-\alpha_{2} v_{1}^{\prime}(0)=b_{1}, & \alpha_{3} v_{1}(1)+\alpha_{4} v_{1}^{\prime}(1)=b_{1}, \\
\alpha_{1} v_{2}(0)-\alpha_{2} v_{2}^{\prime}(0)=b_{2}, & \alpha_{3} v_{2}(1)+\alpha_{4} v_{2}^{\prime}(1)=b_{2} .
\end{array}
$$

Our motivation to study system (1) is that it had made a considerable impact on the study of linear and nonlinear partial differential equations (PDEs), see e.g. [5-10] and the references therein. For instance, if the nonlinear Laplace and heat equations (HEqs) have the stationary solutions, then using appropriate substitutions, these both PDEs are transformed into the second-order nonlinear ordinary differential equations of the type (1), see e.g. [6] and the references therein. Particularly, (1)-(5) models the coupled thermostat phenomena for choosing $\alpha_{3}$ arbitrary, $\alpha_{2}=1=\alpha_{4}$, and $\alpha_{1}=b_{1}=b_{2}=0$. The solution of ordinary differential systems (ODSs) (1) leads to the stationary solution of the system of HEqs, corresponding to a heated bar of length 1 , with a controller at $s=1$, and sensors are being used to provide the feedback to the endpoints where controllers add or remove heat according to the feedback as received by the sensors, see e.g. [11-13] and the references therein.

Another motivation to study (1) is the nonlinear coupled mass-spring phenomena modeled by classical second-order nonlinear coupled systems. System (1) with appropriate boundary conditions (BCs) is used in determining the motion of two springs with weights attached, hung in series from the ceiling, see e.g. [14]. By considering the effects of nonlinearities, viscous damping, restoring forces, and sinusoidal forcing terms, we solve the coupled mass-spring model with Dirichlet type BCs (3) in Sect. 4. 
Another important factor to study the NBVPs is their applicability in modeling various chemical, physical, mechanical, and biological phenomena, see e.g. [15-18] and the references therein. In particular, the nonlinear second-order coupled ODSs have been used in explaining various applied phenomena, such as reaction-diffusion processes, LotkaVolterra models, Sturm-Liouville problems, interaction systems, and some chemical and biological processes, see e.g. [19-24] and the references therein. For studying the applications of NBVPs in engineering and physics, we refer the reader to study Chap. 1 of [25] and [26]. Some works related to nonlinear fractional BVPs for the reader's interest are also listed there (see [27-33] and the references therein).

The applications of NBVPs with generalized NBCs in various areas of physical sciences motivated the researchers towards the development of existence and uniqueness results by employing the lower and upper solutions approach or fixed point index theory in cone. Among them some results are presented as follows.

In [1], the authors developed the new approach based on the idea of coupled lower and upper solutions to study the existence of solutions for the following nonlinear BVPs (notations here are changed from the original papers):

$$
v_{1}^{\prime}(s)=h_{1}\left(s, v_{1}(s)\right), \quad s \in[0, S], S>0,
$$

subject to the following generalized NBCs:

$$
\Upsilon_{1}\left(v_{1}(0), v_{1}(S)\right)
$$

In [2], the authors developed a generalized technique to unify the existence criteria for certain BVPs which have been previously studied on a case-by-case basis. For instance, the initial value problems, the BVPs, and anti-periodic BVPs were the special cases. In particular, the following BVPs were considered (notations here are changed from the original papers):

$$
v_{1}^{\prime}(s)=H_{1}\left(s, v_{1}(s)\right), \quad s \in[0, S], S>0,
$$

subject to the following generalized NBCs:

$$
\Upsilon_{1}\left(v_{1}(0), v_{1}(S)\right)
$$

The approach used in [2] was topological in nature, demonstrated in the form of differential inequalities (lower and upper solutions, coupled lower and upper solutions).

The results of [2] and [1] were extended in [3] to the second-order ordinary NBVPs with the generalized NBCs.

In [4], the authors investigated the existence results for the following first-order nonlinear coupled ODSs (notations here are changed from the original papers):

$$
\begin{array}{ll}
v_{1}^{\prime}(s)=h_{1}\left(s, v_{2}(s)\right), & s \in[0,1], \\
v_{2}^{\prime}(s)=h_{2}\left(s, v_{1}(s)\right), & s \in[0,1],
\end{array}
$$


subject to the following generalized NBCs:

$$
\Upsilon_{1}\left(v_{1}(0), v_{2}(0), v_{1}(1), v_{2}(1)\right)
$$

The tools used in [4] were the coupled lower and upper solutions (LUSs) approach and some results of classical fixed point theory.

The approach was further extended in [34] to second-order nonlinear coupled ODSs with generalized NBCs. One of the interests in this paper was the unification of the existence criteria of certain NBVPs which have been previously treated on a case-by-case basis.

In [35], the authors developed the existence and localization results to study the existence of solutions for the following fully nonlinear coupled ODSs (notations here are changed from the original paper):

$$
v_{i}^{\prime}(s)=h_{i}\left(s, v_{1}(s), v_{2}(s), \ldots, v_{n}(s)\right), \quad i=1,2, \ldots, n, s \in[a, b],
$$

subject to the following nonlinear functional BCs:

$$
\Upsilon_{i}(a)=\Upsilon_{i}(b), \quad i=1,2, \ldots, n .
$$

The approach used in [35] is topological in nature and demonstrated in the forms of differential inequalities (upper and lower solutions).

In [7], the authors studied the existence of multiplicity of nonzero radial solutions for the class of elliptic BVPs with the composition of nonlocal BCs by considering the following coupled system corresponding to elliptic BVPs:

$$
\begin{array}{ll}
v_{1}^{\prime \prime}(s)=g_{1}(s) h_{1}\left(s, v_{1}(s), v_{2}(s)\right), & \text { a.e. on }[0,1], \\
v_{2}^{\prime \prime}(s)=g_{2}(s) h_{2}\left(s, v_{1}(s), v_{2}(s)\right), & \text { a.e. on }[0,1],
\end{array}
$$

subject to the following nonlocal BCs:

$$
\begin{array}{lll}
v_{1}^{\prime}(0)=0, & \beta_{1} v_{1}\left(\alpha_{1}\right)=v_{1}(1), & 0<\alpha_{1}<1, \\
v_{2}^{\prime}(0)=0, & \beta_{2} v_{2}^{\prime}\left(\alpha_{2}\right)=v_{1}(1), & 0<\alpha_{2}<1 .
\end{array}
$$

The approach used in [7] was topological coupled with fixed point index theory. The space involved in this paper did not allow the derivative dependence nonlinearities.

In [8], the authors studied the existence of positive solutions for the following nonlinear second-order coupled ODSs:

$$
\begin{array}{ll}
-v_{1}^{\prime \prime}(s)=h_{1}\left(s, v_{1}(s), v_{2}(s), v_{1}^{\prime}(s), v_{2}^{\prime}(s)\right), & s \in(0,1), \\
-v_{2}^{\prime \prime}(s)=h_{2}\left(s, v_{1}(s), v_{2}(s), v_{1}^{\prime}(s), v_{2}^{\prime}(s)\right), & s \in(0,1),
\end{array}
$$

subject to the following BCs:

$$
v_{1}(0)=v_{1}(1)=v_{2}(0)=v_{2}(1)=0 \text {. }
$$


The tools used in [8] were topological and integral equations. As a byproduct, considering the suitable assumptions, the following elliptic system equivalent to system (6) was solved in the same paper:

$$
\begin{array}{ll}
-\Delta v_{1}=h_{1}\left(|s|, v_{1}, v_{2},\left|\nabla v_{1}\right|,\left|\nabla v_{2}\right|\right), & \text { in } D, \\
-\Delta v_{2}=h_{2}\left(|s|, v_{1}, v_{2},\left|\nabla v_{1}\right|,\left|\nabla v_{2}\right|\right), & \text { in } D,
\end{array}
$$

subject to the following Dirichlet BCs:

$$
v_{1}=v_{2}=0 \quad \text { on } \partial D,
$$

where $D=\left\{s: r_{1}<|s|<r_{2}\right\}$ is an annular domain and $h_{1}, h_{2}$ are continuous functions. Recently, the authors solved system (7) with Neumann BCs and found its radial solution. In fact, the radial solution of (7) is the solution of the following second-order nonlinear coupled ODSs, see e.g. [9] and the references therein:

$$
\begin{array}{ll}
-v_{1}^{\prime \prime}(s)+v_{1}^{2} v_{1}(s)=h_{1}\left(s, v_{1}(s), v_{2}(s),\left|v_{1}^{\prime}(s)\right|,\left|v_{2}^{\prime}(s)\right|\right), & s \in[0,1], \\
-v_{2}^{\prime \prime}(s)+v_{2}^{2} v_{2}(s)=h_{2}\left(s, v_{1}(s), v_{2}(s),\left|v_{1}^{\prime}(s)\right|,\left|v_{2}^{\prime}(s)\right|\right), & s \in[0,1],
\end{array}
$$

subject to the following Neumann BCs:

$$
v_{1}^{\prime}(0)=v_{1}^{\prime}(1)=v_{2}^{\prime}(0)=v_{2}^{\prime}(1)=0 .
$$

Motivated by the aforementioned studies, we extend the results of [1-4] to a generalized class of coupled NBCs and ensure the existence of at least one solution of coupled system (1) subject to generalized BCs (2) by finding the fixed points of the appropriate operator in the space $C^{1}[0,1] \times C^{1}[0,1]$.

The arguments we use in this work are the coupled LUSs approach, Nagumo conditions, Schauder's fixed point and Arzelà-Ascoli theorems.

The classical coupled LUSs approach guarantees the a priori bounds on the solutions of NBVPs, but to get the a priori bounds on the derivatives of the solution functions, the authors used a variety of conditions including guiding functions, barrier strips, BernsteinNagumo quadratic growth conditions, and simply Nagumo conditions, see e.g. [36-44] and the references therein. In this paper, we use the Nagumo conditions.

The rest of the article is structured as follows.

In Sect. 2, some preliminary results and definitions are recalled. In Sect. 3, a generalized result is presented to ensure the existence of solutions of (1)-(2). One of the advantages of this result is to unify the classical existence results of Neumann and Dirichlet BVPs which have previously been studied separately. In Sect. 4, two examples are taken to demonstrate how the results of Sect. 3 can be applied, and the new results clearly demonstrate the advancement made over current literature. In particular, the coupled mass-spring model with Dirichlet type BCs is solved in this section. 


\section{Preliminaries}

Definition 1 The couple of functions $\left(\gamma_{1}, \gamma_{2}\right) \in C^{2}[0,1] \times C^{2}[0,1]$ are said to be the lower solutions of (1) if

$$
\begin{array}{ll}
-\gamma_{1}^{\prime \prime}(s) \leq h_{1}\left(s, \gamma_{1}(s), \gamma_{2}(s), \gamma_{1}^{\prime}(s), \gamma_{2}^{\prime}(s)\right), & s \in[0,1], \\
-\gamma_{2}^{\prime \prime}(s) \leq h_{2}\left(s, \gamma_{1}(s), \gamma_{2}(s), \gamma_{1}^{\prime}(s), \gamma_{2}^{\prime}(s)\right), & s \in[0,1] .
\end{array}
$$

On the same fashion, the couple of functions $\left(\xi_{1}, \xi_{2}\right) \in C^{2}[0,1] \times C^{2}[0,1]$ are said to be the upper solutions of (1) if (8) is satisfied in reverse order.

If $u(s) \leq v(s), \forall s \in[0,1]$, then we may write $u \leq v$, and define the following set:

$$
[u, v]=\left\{x \in C^{1}[0,1]: u(s) \leq x(s) \leq v(s), s \in[0,1]\right\} .
$$

Throughout our paper, we use $\preceq$ as

$$
(x, y) \preceq(w, z) \quad \Longrightarrow \quad x \leq w \text { and } y \leq z .
$$

Next, we define the Nagumo conditions.

Definition 2 Suppose that $\gamma_{1}(s), \gamma_{2}(s), \xi_{1}(s)$, and $\xi_{2}(s)$ are continuous functions such that

$$
\left(\gamma_{1}(s), \gamma_{2}(s)\right) \preceq\left(\xi_{1}(s), \xi_{2}(s)\right), \quad \forall s \in[0,1] .
$$

The continuous functions $h_{1}, h_{2}:[0,1] \times \mathbb{R}^{4} \rightarrow \mathbb{R}$ satisfy a Nagumo condition relative to the intervals $\left[\gamma_{1}(s), \xi_{1}(s)\right]$ and $\left[\gamma_{2}(s), \xi_{2}(s)\right]$ if, for

$$
\begin{aligned}
& P_{1}:=\max \left\{\xi_{1}(1)-\gamma_{1}(0), \xi_{1}(0)-\gamma_{1}(1)\right\}, \\
& P_{2}:=\max \left\{\xi_{2}(1)-\gamma_{2}(0), \xi_{2}(0)-\gamma_{2}(1)\right\},
\end{aligned}
$$

there exist $G_{1}, G_{2}$ constants such that

$$
\begin{aligned}
& G_{1}>\left\{P_{1}, \sup _{s \in[0,1]}\left|\gamma_{1}^{\prime}(s)\right|, \sup _{s \in[0,1]}\left|\xi_{1}^{\prime}(s)\right|\right\}, \\
& G_{2}>\left\{P_{2}, \sup _{s \in[0,1]}\left|\gamma_{2}^{\prime}(s)\right|, \sup _{s \in[0,1]}\left|\xi_{2}^{\prime}(s)\right|\right\},
\end{aligned}
$$

and continuous functions $g_{1}, g_{2}:[0, \infty) \rightarrow[0, \infty)$ such that

$$
\left(\left|h_{1}(s, x, y, z, w)\right|,\left|h_{2}(s, x, y, z, w)\right|\right) \preceq\left(g_{1}(|z|), g_{2}(|w|)\right),
$$

and for

$$
\left(\gamma_{1}(s), \gamma_{2}(s)\right) \preceq(x, y) \preceq\left(\xi_{1}(s), \xi_{2}(s)\right), \quad \forall s \in[0,1],
$$

verifying

$$
\left(\int_{P_{1}}^{G_{1}} \frac{\mathrm{d} t}{g_{1}(t)}, \int_{P_{2}}^{G_{2}} \frac{\mathrm{d} t}{g_{2}(t)}\right) \succ(1,1) .
$$


Now define the following important lemma which is very useful to justify Claim 1 of Theorem 1.

Lemma 1 Let $\Gamma: C^{1}[0,1] \times C^{1}[0,1] \rightarrow C_{0}[0,1] \times C_{0}[0,1] \times \mathbb{R}^{2} \times \mathbb{R}^{2}$ be defined by

$$
\begin{aligned}
\Gamma\left(v_{1}, v_{2}\right)(s)= & \left(v_{1}^{\prime}(s)-v_{1}^{\prime}(0)-\psi \int_{0}^{s} v_{1}(t) \mathrm{d} t, v_{2}^{\prime}(s)-v_{2}^{\prime}(0)-\psi \int_{0}^{s} v_{2}(t) \mathrm{d} t,\right. \\
& \left(\varpi_{1} v_{1}(0)+\varpi_{2} v_{1}(1), \varpi_{3} v_{2}(0)+\varpi_{4} v_{2}(1)\right), \\
& \left.\left(\varpi_{1}^{\prime} v_{1}(0)+\varpi_{2}^{\prime} v_{1}(1), \varpi_{3}^{\prime} v_{2}(0)+\varpi_{4}^{\prime} v_{2}(1)\right)\right),
\end{aligned}
$$

where $\varpi_{1}, \varpi_{2}, \varpi_{3}, \varpi_{4}, \varpi_{1}^{\prime}, \varpi_{2}^{\prime}, \varpi_{3}^{\prime}, \varpi_{4}^{\prime} \in \mathbb{R}$ with $\psi>0$, such that

$$
\left(\varpi_{1} \varpi_{4}-\varpi_{2} \varpi_{3}\right)\left(\varpi_{1}^{\prime} \varpi_{4}^{\prime}-\varpi_{2}^{\prime} \varpi_{3}^{\prime}\right)\left(e^{-\sqrt{\psi}}-e^{\sqrt{\psi}}\right) \neq 0,
$$

and here

$$
C_{0}[0,1]=\{x \in C[0,1]: x(0)=0\} .
$$

Then $\Gamma^{-1}$ exists, and it can be expressed as

$$
\begin{aligned}
\Gamma^{-1}(w, z,(\zeta, \eta),(v, \rho))= & \left(\chi_{1} e^{\sqrt{\psi s}}+\chi_{2} e^{-\sqrt{\psi s}}+\frac{1}{2} \int_{0}^{s} e^{\sqrt{\psi}(s-t)} w(t) \mathrm{d} t\right. \\
& +\frac{1}{2} \int_{0}^{s} e^{\sqrt{\psi(t-s)}} w(t) \mathrm{d} t, \chi_{3} e^{\sqrt{\psi s}}+\chi_{4} e^{-\sqrt{\psi s}} \\
& \left.+\frac{1}{2} \int_{0}^{s} e^{\sqrt{\psi(s-t)}} z(t) \mathrm{d} t+\frac{1}{2} \int_{0}^{s} e^{\sqrt{\psi}(t-s)} z(t) \mathrm{d} t\right),
\end{aligned}
$$

with

$$
\begin{aligned}
\chi_{1}= & \frac{1}{\left(\varpi_{1} \varpi_{4}-\varpi_{2} \varpi_{3}\right)\left(e^{\sqrt{\psi}}-e^{-\sqrt{\psi}}\right)}\left(2 \eta\left(\varpi_{1}+\varpi_{2} e^{-\sqrt{\psi}}\right)-\varpi_{4}\left(\varpi_{1}+\varpi_{2} e^{-\sqrt{\psi}}\right)\right. \\
& \int_{0}^{1} e^{\sqrt{\psi}(1-t)} w(t) \mathrm{d} t+\varpi_{4}\left(\varpi_{1}+\varpi_{2} e^{-\sqrt{\psi}}\right) \int_{0}^{1} e^{\sqrt{\psi}(t-1)} w(t) \mathrm{d} t \\
& -2 \zeta\left(\varpi_{3}+\varpi_{4} e^{-\sqrt{\psi}}\right)+\varpi_{2}\left(\varpi_{2}+\varpi_{3} e^{-\sqrt{\psi}}\right) \int_{0}^{1} e^{\sqrt{\psi}(1-t)} w(t) \mathrm{d} t \\
& \left.-\varpi_{2}\left(\varpi_{3}+\varpi_{4} e^{-\sqrt{\psi}}\right) \int_{0}^{1} e^{\sqrt{\psi}(t-1)} w(t) \mathrm{d} t\right) \\
\chi_{2}= & \frac{1}{\left(\varpi_{1} \varpi_{4}-\varpi_{2} \varpi_{3}\right)\left(e^{-\sqrt{\psi}}-e^{\sqrt{\psi}}\right)}\left(2 \eta\left(\varpi_{1}+\varpi_{2} e^{\sqrt{\psi}}\right)-\varpi_{4}\left(\varpi_{1}+\varpi_{2} e^{\sqrt{\psi}}\right)\right. \\
& \int_{0}^{1} e^{\sqrt{\psi}(1-t)} w(t) \mathrm{d} t+\varpi_{4}\left(\varpi_{1}+\varpi_{2} e^{\sqrt{\psi}}\right) \int_{0}^{1} e^{\sqrt{\psi}(t-1)} w(t) \mathrm{d} t \\
& -2 \zeta\left(\varpi_{3}+\varpi_{4} e^{\sqrt{\psi}}\right)+\varpi_{2}\left(\varpi_{2}+\varpi_{3} e^{\sqrt{\psi}}\right) \int_{0}^{1} e^{\sqrt{\psi}(1-t)} w(t) \mathrm{d} t \\
& \left.-\varpi_{2}\left(\varpi_{3}+\varpi_{4} e^{\sqrt{\psi}}\right) \int_{0}^{1} e^{\sqrt{\psi}(t-1)} w(t) \mathrm{d} t\right)
\end{aligned}
$$




$$
\begin{aligned}
\chi_{3}= & \frac{1}{\left(\varpi_{1}^{\prime} \varpi_{4}^{\prime}-\varpi_{2}^{\prime} \varpi_{3}^{\prime}\right)\left(e^{\sqrt{\psi}}-e^{-\sqrt{\psi}}\right)}\left(2 \rho\left(\varpi_{1}^{\prime}+\varpi_{2}^{\prime} e^{-\sqrt{\psi}}\right)-\varpi_{4}^{\prime}\left(\varpi_{1}^{\prime}+\varpi_{2}^{\prime} e^{-\sqrt{\psi}}\right)\right. \\
& \int_{0}^{1} e^{\sqrt{\psi}(1-t)} z(t) \mathrm{d} t+\varpi_{2}^{\prime}\left(\varpi_{1}^{\prime}+\varpi_{2}^{\prime} e^{-\sqrt{\psi}}\right) \int_{0}^{1} e^{\sqrt{\psi}(t-1)} z(t) \mathrm{d} t \\
& -2 v\left(\varpi_{3}^{\prime}+\varpi_{4}^{\prime} e^{-\sqrt{\psi}}\right)+\varpi_{2}^{\prime}\left(\varpi_{3}^{\prime}+\varpi_{4}^{\prime} e^{-\sqrt{\psi}}\right) \int_{0}^{1} e^{\sqrt{\psi}(1-t)} z(t) \mathrm{d} t \\
& \left.-\varpi_{2}^{\prime}\left(\varpi_{3}^{\prime}+\varpi_{4}^{\prime} e^{-\sqrt{\psi}}\right) \int_{0}^{1} e^{\sqrt{\psi}(t-1)} z(t) \mathrm{d} t\right)
\end{aligned}
$$

and

$$
\begin{aligned}
\chi_{4}= & \frac{1}{\left(\varpi_{1}^{\prime} \varpi_{4}^{\prime}-\varpi_{2}^{\prime} \varpi_{3}^{\prime}\right)\left(e^{-\sqrt{\psi}}-e^{\sqrt{\psi}}\right)}\left(2 \rho\left(\varpi_{1}^{\prime}+\varpi_{2}^{\prime} e^{\sqrt{\psi}}\right)-\varpi_{4}^{\prime}\left(\varpi_{1}^{\prime}+\varpi_{2}^{\prime} e^{\sqrt{\psi}}\right)\right. \\
& \int_{0}^{1} e^{\sqrt{\psi}(1-t)} z(t) \mathrm{d} t+\varpi_{2}^{\prime}\left(\varpi_{1}^{\prime}+\varpi_{2}^{\prime} e^{\sqrt{\psi}}\right) \int_{0}^{1} e^{\sqrt{\psi}(t-1)} z(t) \mathrm{d} t \\
& -2 v\left(\varpi_{3}^{\prime}+\varpi_{4}^{\prime} e^{\sqrt{\psi}}\right)+\varpi_{2}^{\prime}\left(\varpi_{3}^{\prime}+\varpi_{4}^{\prime} e^{\sqrt{\psi}}\right) \int_{0}^{1} e^{\sqrt{\psi}(1-t)} z(t) \mathrm{d} t \\
& \left.-\varpi_{2}^{\prime}\left(\varpi_{3}^{\prime}+\varpi_{4}^{\prime} e^{\sqrt{\psi}}\right) \int_{0}^{1} e^{\sqrt{\psi}(t-1)} z(t) \mathrm{d} t\right) .
\end{aligned}
$$

Proof Choose $w, z \in C_{0}[0,1]$ and $\zeta, \eta, \nu, \rho \in \mathbb{R}$ as

$$
\begin{aligned}
& w(s)=v_{1}^{\prime}(s)-v_{1}^{\prime}(0)-\psi \int_{0}^{s} v_{1}(t) \mathrm{d} t, \\
& z(s)=v_{2}^{\prime}(s)-v_{2}^{\prime}(0)-\psi \int_{0}^{s} v_{2}(t) \mathrm{d} t, \\
& \zeta=\varpi_{1} v_{1}(0)+\varpi_{2} v_{1}(1), \\
& \eta=\varpi_{3} v_{2}(0)+\varpi_{4} v_{2}(1), \\
& v=\varpi_{1}^{\prime} v_{1}(0)+\varpi_{2}^{\prime} v_{1}(1), \\
& \rho=\varpi_{3}^{\prime} v_{2}(0)+\varpi_{4}^{\prime} v_{2}(1) .
\end{aligned}
$$

In the light of (22)-(27), (16) can also be written as

$$
\Gamma\left(v_{1}, v_{2}\right)(s)=(w(s), z(s),(\zeta, \eta),(v, \rho)) .
$$

On differentiating (22), we have

$$
w^{\prime}(s)=v_{1}^{\prime \prime}(s)-\psi v_{1}(s) .
$$

Solving (29), we have

$$
v_{1}(s)=\chi_{1} e^{\sqrt{\psi} s}+\chi_{2} e^{-\sqrt{\psi s}}+\frac{1}{2} \int_{0}^{s} e^{\sqrt{\psi(s-t)}} w(t) \mathrm{d} t+\frac{1}{2} \int_{0}^{s} e^{\sqrt{\psi(t-s)}} w(t) \mathrm{d} t .
$$


Using (24) and (25), we have the following system of equations:

$$
\begin{aligned}
\zeta= & \left(\varpi_{1}+\varpi_{2} e^{\sqrt{\psi}}\right) \chi_{1}+\left(\varpi_{1}+\varpi_{2} e^{-\sqrt{\psi}}\right) \chi_{2} \\
& +\frac{\varpi_{2}}{2}\left(\int_{0}^{1} e^{\sqrt{\psi}(1-t)} w(t) \mathrm{d} t+e^{\sqrt{\psi}(t-1)} w(t) \mathrm{d} t\right), \\
\eta= & \left(\varpi_{3}+\varpi_{4} e^{\sqrt{\psi}}\right) \chi_{1}+\left(\varpi_{3}+\varpi_{4} e^{-\sqrt{\psi}}\right) \chi_{2} \\
& +\frac{\varpi_{4}}{2}\left(\int_{0}^{1} e^{\sqrt{\psi}(1-t)} w(t) \mathrm{d} t+e^{\sqrt{\psi}(t-1)} w(t) \mathrm{d} t\right) .
\end{aligned}
$$

Solving (31), we have

$$
\begin{aligned}
\chi_{1}= & \frac{1}{\left(\varpi_{1} \varpi_{4}-\varpi_{2} \varpi_{3}\right)\left(e^{\sqrt{\psi}}-e^{-\sqrt{\psi}}\right)}\left(2 \eta\left(\varpi_{1}+\varpi_{2} e^{-\sqrt{\psi}}\right)-\varpi_{4}\left(\varpi_{1}+\varpi_{2} e^{-\sqrt{\psi}}\right)\right. \\
& \int_{0}^{1} e^{\sqrt{\psi}(1-t)} w(t) \mathrm{d} t+\varpi_{4}\left(\varpi_{1}+\varpi_{2} e^{-\sqrt{\psi}}\right) \int_{0}^{1} e^{\sqrt{\psi}(t-1)} w(t) \mathrm{d} t \\
& -2 \zeta\left(\varpi_{3}+\varpi_{4} e^{-\sqrt{\psi}}\right)+\varpi_{2}\left(\varpi_{2}+\varpi_{3} e^{-\sqrt{\psi}}\right) \int_{0}^{1} e^{\sqrt{\psi}(1-t)} w(t) \mathrm{d} t \\
& \left.-\varpi_{2}\left(\varpi_{3}+\varpi_{4} e^{-\sqrt{\psi}}\right) \int_{0}^{1} e^{\sqrt{\psi}(t-1)} w(t) \mathrm{d} t\right) \\
\chi_{2}= & \left(\varpi_{1} \varpi_{4}-\varpi_{2} \varpi_{3}\right)\left(e^{-\sqrt{\psi}}-e^{\sqrt{\psi})}\left(2 \eta\left(\varpi_{1}+\varpi_{2} e^{\sqrt{\psi}}\right)-\varpi_{4}\left(\varpi_{1}+\varpi_{2} e^{\sqrt{\psi}}\right)\right.\right. \\
& \int_{0}^{1} e^{\sqrt{\psi}(1-t)} w(t) \mathrm{d} t+\varpi_{4}\left(\varpi_{1}+\varpi_{2} e^{\sqrt{\psi}}\right) \int_{0}^{1} e^{\sqrt{\psi}(t-1)} w(t) \mathrm{d} t \\
& -2 \zeta\left(\varpi_{3}+\varpi_{4} e^{\sqrt{\psi}}\right)+\varpi_{2}\left(\varpi_{2}+\varpi_{3} e^{\sqrt{\psi}}\right) \int_{0}^{1} e^{\sqrt{\psi}(1-t)} w(t) \mathrm{d} t \\
& \left.-\varpi_{2}\left(\varpi_{3}+\varpi_{4} e^{\sqrt{\psi}}\right) \int_{0}^{1} e^{\sqrt{\psi}(t-1)} w(t) \mathrm{d} t\right) .
\end{aligned}
$$

Similarly, we can show the following:

$$
v_{2}(s)=\chi_{3} e^{\sqrt{\psi} s}+\chi_{4} e^{-\sqrt{\psi} s}+\frac{1}{2} \int_{0}^{s} e^{\sqrt{\psi}(s-t)} z(t) \mathrm{d} t+\frac{1}{2} \int_{0}^{s} e^{\sqrt{\psi}(t-s)} z(t) \mathrm{d} t
$$

with

$$
\begin{aligned}
\chi_{3}= & \frac{1}{\left(\varpi_{1}^{\prime} \varpi_{4}^{\prime}-\varpi_{2}^{\prime} \varpi_{3}^{\prime}\right)\left(e^{\sqrt{\psi}}-e^{-\sqrt{\psi}}\right)}\left(2 \rho\left(\varpi_{1}^{\prime}+\varpi_{2}^{\prime} e^{-\sqrt{\psi}}\right)-\varpi_{4}^{\prime}\left(\varpi_{1}^{\prime}+\varpi_{2}^{\prime} e^{-\sqrt{\psi}}\right)\right. \\
& \int_{0}^{1} e^{\sqrt{\psi}(1-t)} z(t) \mathrm{d} t+\varpi_{2}^{\prime}\left(\varpi_{1}^{\prime}+\varpi_{2}^{\prime} e^{-\sqrt{\psi}}\right) \int_{0}^{1} e^{\sqrt{\psi}(t-1)} z(t) \mathrm{d} t \\
& -2 v\left(\varpi_{3}^{\prime}+\varpi_{4}^{\prime} e^{-\sqrt{\psi}}\right)+\varpi_{2}^{\prime}\left(\varpi_{3}^{\prime}+\varpi_{4}^{\prime} e^{-\sqrt{\psi}}\right) \int_{0}^{1} e^{\sqrt{\psi}(1-t)} z(t) \mathrm{d} t \\
& \left.-\varpi_{2}^{\prime}\left(\varpi_{3}^{\prime}+\varpi_{4}^{\prime} e^{-\sqrt{\psi}}\right) \int_{0}^{1} e^{\sqrt{\psi}(t-1)} z(t) \mathrm{d} t\right)
\end{aligned}
$$


and

$$
\begin{aligned}
\chi_{4}= & \frac{1}{\left(\varpi_{1}^{\prime} \varpi_{4}^{\prime}-\varpi_{2}^{\prime} \varpi_{3}^{\prime}\right)\left(e^{-\sqrt{\psi}}-e^{\sqrt{\psi}}\right)}\left(2 \rho\left(\varpi_{1}^{\prime}+\varpi_{2}^{\prime} e^{\sqrt{\psi}}\right)-\varpi_{4}^{\prime}\left(\varpi_{1}^{\prime}+\varpi_{2}^{\prime} e^{\sqrt{\psi}}\right)\right. \\
& \int_{0}^{1} e^{\sqrt{\psi}(1-t)} z(t) \mathrm{d} t+\varpi_{2}^{\prime}\left(\varpi_{1}^{\prime}+\varpi_{2}^{\prime} e^{\sqrt{\psi}}\right) \int_{0}^{1} e^{\sqrt{\psi(t-1)}} z(t) \mathrm{d} t \\
& -2 v\left(\varpi_{3}^{\prime}+\varpi_{4}^{\prime} e^{\sqrt{\psi}}\right)+\varpi_{2}^{\prime}\left(\varpi_{3}^{\prime}+\varpi_{4}^{\prime} e^{\sqrt{\psi}}\right) \int_{0}^{1} e^{\sqrt{\psi}(1-t)} z(t) \mathrm{d} t \\
& \left.-\varpi_{2}^{\prime}\left(\varpi_{3}^{\prime}+\varpi_{4}^{\prime} e^{\sqrt{\psi}}\right) \int_{0}^{1} e^{\sqrt{\psi(t-1)}} z(t) \mathrm{d} t\right) .
\end{aligned}
$$

Equation (28) can also be written as

$$
\left(v_{1}(s), v_{2}(s)\right)=\Gamma^{-1}(w(s), z(s),(\zeta, \eta),(\nu, \rho)) .
$$

Hence, (30)-(34) prove (17).

Next, we construct the definition of coupled LUSs which is useful in unifying the existence results of certain BVPs which were previously studied separately.

Definition 3 We say that $\left(\gamma_{1}, \gamma_{2}\right),\left(\xi_{1}, \xi_{2}\right) \in C^{2}[0,1] \times C^{2}[0,1]$ are coupled LUSs for (1)-(2) if $\left(\gamma_{1}, \gamma_{2}\right)$ is a lower solution and $\left(\xi_{1}, \xi_{2}\right)$ is an upper solution for $(1)$, such that

$$
\begin{aligned}
& \Upsilon_{1}\left(\xi_{1}(0), \xi_{2}(0), \xi_{1}(1), \xi_{2}(1), \xi_{1}^{\prime}(0), \xi_{2}^{\prime}(0)\right) \\
& \quad \preceq(0,0) \preceq \Upsilon_{1}\left(\gamma_{1}(0), \gamma_{2}(0), \gamma_{1}(1), \gamma_{2}(1), \gamma_{1}^{\prime}(0), \gamma_{2}^{\prime}(0)\right), \\
& \Upsilon_{2}\left(\xi_{1}(0), \xi_{2}(0), \xi_{1}(1), \xi_{2}(1), \xi_{1}^{\prime}(1), \xi_{2}^{\prime}(1)\right) \\
& \quad \preceq(0,0) \preceq \Upsilon_{2}\left(\gamma_{1}(0), \gamma_{2}(0), \gamma_{1}(1), \gamma_{2}(1), \gamma_{1}^{\prime}(1), \gamma_{2}^{\prime}(1)\right), \\
& \Upsilon_{1}\left(\xi_{1}(0), \xi_{2}(0), \gamma_{1}(1), \gamma_{2}(1), \xi_{1}^{\prime}(0), \xi_{2}^{\prime}(0)\right) \\
& \quad \preceq(0,0) \preceq \Upsilon_{1}\left(\gamma_{1}(0), \gamma_{2}(0), \xi_{1}(1), \xi_{2}(1), \gamma_{1}^{\prime}(0), \gamma_{2}^{\prime}(0)\right), \\
& \Upsilon_{2}\left(\xi_{1}(0), \xi_{2}(0), \gamma_{1}(1), \gamma_{2}(1), \gamma_{1}^{\prime}(1), \gamma_{2}^{\prime}(1)\right) \\
& \quad \preceq(0,0) \preceq \Upsilon_{2}\left(\gamma_{1}(0), \gamma_{2}(0), \xi_{1}(1), \xi_{2}(1), \xi_{1}^{\prime}(1), \xi_{2}^{\prime}(1)\right) .
\end{aligned}
$$

Inequalities (35)-(36) verify the following classical existence criteria for Dirichlet BVPs ((1) and (3)):

$$
\begin{array}{ll}
\gamma_{1}(0) \leq \alpha_{1} \leq \xi_{1}(0), & \gamma_{2}(0) \leq \alpha_{2} \leq \xi_{2}(0), \\
\gamma_{1}(1) \leq \alpha_{3} \leq \xi_{1}(1), & \gamma_{2}(1) \leq \alpha_{4} \leq \xi_{2}(1),
\end{array}
$$

and inequalities (37)-(38) verify the following classical existence criteria for Neumann BVPs ((1) and (4)):

$$
\begin{array}{ll}
\xi_{1}^{\prime}(0) \leq \alpha_{1} \leq \gamma_{1}^{\prime}(0), & \xi_{2}^{\prime}(0) \leq \alpha_{2} \leq \gamma_{2}^{\prime}(0), \\
\gamma_{1}^{\prime}(1) \leq \alpha_{3} \leq \xi_{1}^{\prime}(1), & \gamma_{2}^{\prime}(1) \leq \alpha_{4} \leq \xi_{2}^{\prime}(1),
\end{array}
$$

where $\alpha_{1}, \alpha_{2}, \alpha_{3}, \alpha_{4} \in \mathbb{R}$. 


\section{Main result}

The following is the main result of the paper, which unifies the existence criteria of certain BVPs which have been previously treated separately. The arguments we use to prove the main result are the Arzelà-Ascoli theorem and Schauder's fixed point theorem.

Theorem 1 Let system (1)-(2) have $\left(\gamma_{1}, \gamma_{2}\right),\left(\xi_{1}, \xi_{2}\right)$ coupled LUSs and the boundary functions $h_{1}, h_{2}$ verify the Nagumo conditions relative to $\left[\gamma_{1}(s), \xi_{1}(s)\right]$ and $\left[\gamma_{2}(s), \xi_{2}(s)\right]$. Assume that $\Upsilon_{2}$ and $\Upsilon_{1}$ are nonincreasing and nondecreasing in the fifth and sixth arguments, respectively, and

$$
\begin{aligned}
& \Upsilon_{2\left(\gamma_{1}, \gamma_{2}\right)}(x, y):=\Upsilon_{2}\left(x, y, \gamma_{1}(1), \gamma_{2}(1), \gamma_{1}^{\prime}(1), \gamma_{2}^{\prime}(1)\right), \\
& \Upsilon_{2\left(\xi_{1}, \xi_{2}\right)}(x, y):=\Upsilon_{2}\left(x, y, \xi_{1}(1), \xi_{2}(1), \xi_{1}^{\prime}(1), \xi_{2}^{\prime}(1)\right)
\end{aligned}
$$

are monotone on $\left[\gamma_{1}(0), \xi_{1}(0)\right] \times\left[\gamma_{2}(0), \xi_{2}(0)\right]$ and

$$
\begin{aligned}
& \Upsilon_{1\left(\gamma_{1}, \gamma_{2}\right)}(x, y):=\Upsilon_{1}\left(\gamma_{1}(0), \gamma_{2}(0), x, y, \gamma_{1}^{\prime}(0), \gamma_{2}^{\prime}(0)\right), \\
& \Upsilon_{1\left(\xi_{1}, \xi_{2}\right)}(x, y):=\Upsilon_{1}\left(\xi_{1}(0), \xi_{2}(0), x, y, \xi_{1}^{\prime}(0), \xi_{2}^{\prime}(0)\right)
\end{aligned}
$$

are monotone on $\left[\gamma_{1}(1), \xi_{1}(1)\right] \times\left[\gamma_{2}(1), \xi_{2}(1)\right]$.

Then system (1)-(2) has at least one solution $\left(v_{1}, v_{2}\right) \in\left(C^{2}[0,1], \mathbb{R}\right)^{2}$ such that

$$
\left(\gamma_{1}(s), \gamma_{2}(s)\right) \preceq\left(v_{1}(s), v_{2}(s)\right) \preceq\left(\xi_{1}(s), \xi_{2}(s)\right), \quad \forall s \in[0,1],
$$

and

$$
\left(\left\|v_{1}^{\prime}\right\|,\left\|v_{2}^{\prime}\right\|\right) \preceq\left(G_{1}, G_{2}\right),
$$

with $G_{1}$ and $G_{2}$ given by the Nagumo conditions.

Proof Let the following modified problem be associated with problem (1)-(2), with $\psi>0$ :

$$
\begin{aligned}
& -v_{1}^{\prime \prime}(s)+\psi v_{1}(s)=\hat{H}_{1}\left(s, v_{1}(s), v_{2}(s), v_{1}^{\prime}(s), v_{2}^{\prime}(s)\right), \quad s \in[0,1], \\
& -v_{2}^{\prime \prime}(s)+\psi v_{2}(s)=\hat{H}_{2}\left(s, v_{1}(s), v_{2}(s), v_{1}^{\prime}(s), v_{2}^{\prime}(s)\right), \quad s \in[0,1], \\
& \hat{\Upsilon}_{1}\left(v_{1}(0), v_{2}(0), v_{1}(1), v_{2}(1), v_{1}^{\prime}(0), v_{2}^{\prime}(0)\right)=\left(v_{1}(0), v_{2}(0)\right), \\
& \hat{\Upsilon}_{2}\left(v_{1}(0), v_{2}(0), v_{1}(1), v_{2}(1), v_{1}^{\prime}(1), v_{2}^{\prime}(1)\right)=\left(v_{1}(1), v_{2}(1)\right),
\end{aligned}
$$

with

$$
\begin{aligned}
\hat{H}_{1}\left(s, v_{1}(s), v_{2}(s), v_{1}^{\prime}(s), v_{2}^{\prime}(s)\right)= & h_{1}\left(s, \tau_{1}\left(s, v_{1}(s)\right), \tau_{2}\left(s, v_{2}(s)\right),\right. \\
& \left.\sigma_{1}(u), \sigma_{2}(v)\right)+\psi \tau_{1}\left(s, v_{1}(s)\right), \\
\hat{H}_{2}\left(s, v_{1}(s), v_{2}(s), v_{1}^{\prime}(s), v_{2}^{\prime}(s)\right)= & h_{2}\left(s, \tau_{1}\left(s, v_{1}(s)\right), \tau_{2}\left(s, v_{2}(s)\right),\right. \\
& \left.\sigma_{1}(u), \sigma_{2}(v)\right)+\psi \tau_{2}\left(s, v_{2}(s)\right),
\end{aligned}
$$




$$
\begin{aligned}
\hat{\Upsilon}_{1}(e, f, g, j, o, l)= & \left(\tau_{1}(0, e), \tau_{2}(0, f)\right)+\Upsilon_{1}\left(v_{1}(0), v_{2}(0), v_{1}(1),\right. \\
& \left.v_{2}(1), v_{1}^{\prime}(0), v_{2}^{\prime}(0)\right), \\
\hat{\Upsilon}_{2}(e, f, g, j, o, l)= & \left(\tau_{1}(1, g), \tau_{2}(1, j)\right)+\Upsilon_{2}\left(v_{1}(0), v_{2}(0), v_{1}(1),\right. \\
& \left.v_{2}(1), v_{1}^{\prime}(1), v_{2}^{\prime}(1)\right),
\end{aligned}
$$

and for each $i=1,2$,

$$
\begin{aligned}
& \tau_{i}(s, x)= \begin{cases}\xi_{i}(s) & \text { if } x \not \leq \xi_{i}(s), \\
x & \text { if } \gamma_{i}(s) \leq x \leq \xi_{i}(s), \\
\gamma_{i}(s) & \text { if } x \ngtr \gamma_{i}(s),\end{cases} \\
& \sigma_{i}(x)=\max \left\{-G_{i}, \min \left\{x, G_{i}\right\}\right\},
\end{aligned}
$$

Note that if $\left(v_{1}, v_{2}\right) \in\left[\gamma_{1}, \xi_{1}\right] \times\left[\gamma_{2}, \xi_{2}\right]$ is a solution of (39), then $\left(v_{1}, v_{2}\right)$ is a solution of (1)-(2).

Claim 1 The fixed points of the operator $\Gamma^{-1} \hat{E}$ are the solutions of system (39).

Define the mappings

$$
\Gamma, \hat{E}: C^{1}[0,1] \times C^{1}[0,1] \rightarrow C_{0}[0,1] \times C_{0}[0,1] \times \mathbb{R}^{2} \times \mathbb{R}^{2}
$$

by

$$
\begin{aligned}
\Gamma\left(v_{1}, v_{2}\right)(s)= & \left(v_{1}^{\prime}(s)-v_{1}^{\prime}(0)-\psi \int_{0}^{s} v_{1}(t) \mathrm{d} t, v_{2}^{\prime}(s)-v_{2}^{\prime}(0)-\psi \int_{0}^{s} v_{2}(t) \mathrm{d} t\right. \\
& \left.\left(v_{1}(0), v_{2}(0)\right),\left(v_{1}(1), v_{2}(1)\right)\right),
\end{aligned}
$$

and

$$
\begin{aligned}
\hat{E}\left(v_{1}, v_{2}\right)(s)= & \left(\int_{0}^{s} \hat{H}_{1}\left(t, v_{1}(t), v_{2}(t), v_{1}^{\prime}(t), v_{2}^{\prime}(t)\right) \mathrm{d} t, \int_{0}^{s} \hat{H}_{2}\left(t, v_{1}(t), v_{2}(t), v_{1}^{\prime}(t), v_{2}^{\prime}(t)\right) \mathrm{d} t,\right. \\
& \hat{\Upsilon}_{1}\left(v_{1}(0), v_{2}(0), v_{1}(1), v_{2}(1), v_{1}^{\prime}(0), v_{2}^{\prime}(0)\right) \\
& \left.\hat{\Upsilon}_{2}\left(v_{1}(0), v_{2}(0), v_{1}(1), v_{2}(1), v_{1}^{\prime}(1), v_{2}^{\prime}(1)\right)\right) .
\end{aligned}
$$

Now in view of the Arzelà-Ascoli theorem and the definitions of $\hat{H}_{1}, \hat{H}_{2}, \hat{\Upsilon}_{1}$, and $\hat{\Upsilon}_{2}, \hat{E}$ is continuous and compact. Therefore, the class $\left\{\hat{E}\left(v_{1}, v_{2}\right):\left(v_{1}, v_{2}\right) \in C^{1}[0,1] \times C^{1}[0,1]\right\}$ is uniformly bounded and equicontinuous. Consequently, $\hat{E}$ is a compact map. Moreover, $\Gamma^{-1}$ exists and is continuous (see Lemma 1).

The fixed points of the following operator are the solutions of problem (39) which are guaranteed by Schauder's fixed point theorem because the operator is continuous and compact.

$$
\Gamma^{-1} \hat{E}: C^{1}[0,1] \times C^{1}[0,1] \rightarrow C^{1}[0,1] \times C^{1}[0,1] .
$$


Claim 2 If $\left(v_{1}, v_{2}\right)$ is a solution of (39), then $\left(\gamma_{1}(s), \gamma_{2}(s)\right) \preceq\left(v_{1}(s), v_{2}(s)\right) \preceq\left(\xi_{1}(s), \xi_{2}(s)\right), \forall s \in$ $[0,1]$.

Suppose $\left(v_{1}, v_{2}\right) \preceq\left(\xi_{1}, \xi_{2}\right)$. If $\left(v_{1}, v_{2}\right) \npreceq\left(\xi_{1}, \xi_{2}\right)$, then $v_{1} \leq \xi_{1}$ and $v_{2} \leq \xi_{2}$. If $v_{1} \not \leq \xi_{1}$, then $v_{1}-\xi_{1}$ has positive maximum at some $r_{0} \in(0,1)$ such that $\left(v_{1}-\xi_{1}\right)^{\prime}\left(r_{0}\right)=0$ and $\left(v_{1}-\xi_{1}\right)^{\prime \prime}\left(r_{0}\right)<0$. Also, by Definition 2, we have $\left|v_{1}^{\prime}\left(r_{0}\right)\right|=\left|\xi_{1}^{\prime}\left(r_{0}\right)\right|<G_{1}$. Similarly, on the same fashion, if $v_{2} \not \leq \xi_{2}$, then $\left(v_{2}-\xi_{2}\right)^{\prime}\left(r_{0}\right)=0$, and by Definition 2, we have $\left|v_{2}^{\prime}\left(r_{0}\right)\right|=$ $\left|\xi_{2}^{\prime}\left(r_{0}\right)\right|<G_{2}$. But

$$
\begin{aligned}
\left(v_{1}-\xi_{1}\right)^{\prime \prime}\left(r_{0}\right)> & -\hat{H}_{1}\left(r_{0}, v_{1}\left(r_{0}\right), v_{2}\left(r_{0}\right), v_{1}^{\prime}\left(r_{0}\right), v_{2}^{\prime}\left(r_{0}\right)\right)+\psi v_{1}\left(r_{0}\right) \\
& +h_{1}\left(r_{0}, \xi_{1}\left(r_{0}\right), \xi_{2}\left(r_{0}\right), \xi_{1}^{\prime}\left(r_{0}\right), \xi_{2}^{\prime}\left(r_{0}\right)\right) \\
= & -h_{1}\left(r_{0}, \xi_{1}\left(r_{0}\right), \xi_{2}\left(r_{0}\right), v_{1}^{\prime}\left(r_{0}\right), v_{2}^{\prime}\left(r_{0}\right)\right)-\psi \xi_{1}\left(r_{0}\right)+\psi v_{1}\left(r_{0}\right) \\
& +h_{1}\left(r_{0}, \xi_{1}\left(r_{0}\right), \xi_{2}\left(r_{0}\right), \xi_{1}^{\prime}\left(r_{0}\right), \xi_{2}^{\prime}\left(r_{0}\right)\right) \\
= & -h_{1}\left(r_{0}, \xi_{1}\left(r_{0}\right), \xi_{2}\left(r_{0}\right), \xi_{1}^{\prime}\left(r_{0}\right), \xi_{2}^{\prime}\left(r_{0}\right)\right)-\psi \xi_{1}\left(r_{0}\right)+\psi v_{1}\left(r_{0}\right) \\
& +h_{1}\left(r_{0}, \xi_{1}\left(r_{0}\right), \xi_{2}\left(r_{0}\right), \xi_{1}^{\prime}\left(r_{0}\right), \xi_{2}^{\prime}\left(r_{0}\right)\right) \\
= & \psi\left(v_{1}\left(r_{0}\right)-\xi_{1}\left(r_{0}\right)\right)>0,
\end{aligned}
$$

a contradiction. Similarly, we can show that $\left(\gamma_{1}, \gamma_{2}\right) \preceq\left(v_{1}, v_{2}\right)$. Hence $\left(\gamma_{1}, \gamma_{2}\right) \preceq\left(v_{1}, v_{2}\right) \preceq$ $\left(\xi_{1}, \xi_{2}\right)$

Claim 3 If $\left(v_{1}, v_{2}\right)$ is a solution of (39), then $\left(v_{1}, v_{2}\right)$ must satisfy (2).

For this, we have to show that

$$
\begin{aligned}
\left(\gamma_{1}(0), \gamma_{2}(0)\right) & \preceq\left(v_{1}(0), v_{2}(0)\right)+\Upsilon_{1}\left(v_{1}(0), v_{2}(0), v_{1}(1), v_{2}(1), v_{1}^{\prime}(0), v_{2}^{\prime}(0)\right) \\
& \preceq\left(\xi_{1}(0), \xi_{2}(0)\right) .
\end{aligned}
$$

Using (39), we have

$$
\begin{aligned}
\left(v_{1}(0), v_{2}(0)\right) & =\hat{\Upsilon}_{1}\left(v_{1}(0), v_{2}(0), v_{1}(1), v_{2}(1), v_{1}^{\prime}(0), v_{2}^{\prime}(0)\right) \\
& \left.=\left(\tau_{1}\left(0, v_{1}(0)\right), \tau_{2}\left(0, v_{2}(0)\right)\right)+\Upsilon_{1}\left(v_{1}(0), v_{2}(0), v_{1}(1), v_{2}(1), v_{1}^{\prime}(0), v_{2}^{\prime}(0)\right)\right) \\
& =\left(\xi_{1}(0), \xi_{2}(0)\right) .
\end{aligned}
$$

From Claim 2, we know that $\left(v_{1}, v_{2}\right) \preceq\left(\xi_{1}, \xi_{2}\right)$, and this together with $\left(v_{1}-\xi_{1}, v_{2}-\xi_{2}\right) \in$ $C^{2}[0,1] \times C^{2}[0,1]$ and $\left(v_{1}(0), v_{2}(0)\right)=\left(\xi_{1}(0), \xi_{2}(0)\right)$ yields $v_{1}^{\prime}(0) \leq \xi_{1}^{\prime}(0)$ and $v_{2}^{\prime}(0) \leq \xi_{2}^{\prime}(0)$.

We will prove (40) by contradiction, so assume

$$
\left(v_{1}(0), v_{2}(0)\right)+\Upsilon_{1}\left(v_{1}(0), v_{2}(0), v_{1}(1), v_{2}(1), v_{1}^{\prime}(0), v_{2}^{\prime}(0)\right) \npreceq\left(\xi_{1}(0), \xi_{2}(0)\right) .
$$

If $\Upsilon_{1\left(\xi_{1}, \xi_{2}\right)}(x, y)$ is monotone nonincreasing, then we have

$$
\begin{aligned}
& \left(v_{1}(0), v_{2}(0)\right)+\Upsilon_{1}\left(v_{1}(0), v_{2}(0), v_{1}(1), v_{2}(1), v_{1}^{\prime}(0), v_{2}^{\prime}(0)\right) \\
& \quad=\left(\xi_{1}(0), \xi_{2}(0)\right)+\Upsilon_{1}\left(\xi_{1}(0), \xi_{2}(0), v_{1}(1), v_{2}(1), v_{1}^{\prime}(0), v_{2}^{\prime}(0)\right)
\end{aligned}
$$




$$
\begin{aligned}
& \preceq\left(\xi_{1}(0), \xi_{2}(0)\right)+\Upsilon_{1}\left(\xi_{1}(0), \xi_{2}(0), v_{1}(1), v_{2}(1), \xi_{1}^{\prime}(0), \xi_{2}^{\prime}(0)\right) \\
& =\left(\xi_{1}(0), \xi_{2}(0)\right)+\Upsilon_{1\left(\xi_{1}, \xi_{2}\right)}\left(v_{1}(1), v_{2}(1)\right) \\
& \preceq\left(\xi_{1}(0), \xi_{2}(0)\right)+\Upsilon_{1\left(\xi_{1}, \xi_{2}\right)}\left(\gamma_{1}(1), \gamma_{2}(1)\right) \\
& =\left(\xi_{1}(0), \xi_{2}(0)\right)+\Upsilon_{1}\left(\xi_{1}(0), \xi_{2}(0), \gamma_{1}(1), \gamma_{2}(1), \xi_{1}^{\prime}(0), \xi_{2}^{\prime}(0)\right) \\
& \preceq\left(\xi_{1}(0), \xi_{2}(0)\right),
\end{aligned}
$$

a contradiction. Similarly, if $\Upsilon_{1\left(\xi_{1}, \xi_{2}\right)}(x, y)$ is monotone nondecreasing, then we get the same contradiction. Consequently, (40) holds. Similar reasoning shows the other boundary condition. Consequently, $\left(v_{1}, v_{2}\right)$ satisfies (2). Hence system (1)-(2) has a solution satisfying $\left(\gamma_{1}, \gamma_{2}\right) \preceq\left(v_{1}, v_{2}\right) \preceq\left(\xi_{1}, \xi_{2}\right)$.

Claim 4 Every solution of (39) satisfies

$$
\left\|v_{1}^{\prime}\right\| \leq G_{1} \quad \text { and } \quad\left\|v_{2}^{\prime}\right\| \leq G_{2}
$$

To prove (44), assume that $\left(v_{1}(s), v_{2}(s)\right)$ is a solution of (39) such that

$$
\left(\gamma_{1}(s), \gamma_{2}(s)\right) \preceq\left(v_{1}(s), v_{2}(s)\right) \preceq\left(\xi_{1}(s), \xi_{2}(s)\right), \quad \forall s \in[0,1]
$$

then by the Lagrange theorem, there exists $s_{0}, s_{1} \in[0,1]$ such that

$$
v_{1}^{\prime}\left(s_{0}\right)=v_{1}(1)-v_{1}(0) \quad \text { and } \quad v_{2}^{\prime}\left(s_{1}\right)=v_{2}(1)-v_{2}(0)
$$

Suppose on the contrary that $v_{1}^{\prime}(s)>p_{1}, \forall s \in[0,1]$, then using (45), the following contradiction is obtained:

$$
v_{1}^{\prime}\left(s_{0}\right)=v_{1}(1)-v_{1}(0) \leq \xi_{1}(1)-\gamma_{1}(0) \leq p_{1} .
$$

The similar contradiction is obtained for $v_{1}^{\prime}(s)<-p_{1}, \forall s \in[0,1]$. In the case where $\left|v_{1}^{\prime}(s)\right| \leq$ $p_{1}$, Claim 4 is finished. So, assume that there are $s_{2}, s_{3} \in[0,1]$ with $s_{2}<s_{3}$ such that

$$
v_{1}^{\prime}\left(s_{2}\right)<p_{1} \quad \text { and } \quad v_{1}^{\prime}\left(s_{3}\right)>p_{1} .
$$

By continuing in the same way, there is $s_{4} \in\left[s_{2}, s_{3}\right]$ such that

$$
\left.v_{1}^{\prime}\left(s_{4}\right)=p_{1} \quad \text { and } \quad v_{1}^{\prime}\left(s_{3}\right)>p_{1}, \quad \forall s \in\right] s_{4}, s_{3}[.
$$

So, by a convenient change of variable, by (13) and (15), we have

$$
\begin{aligned}
\int_{v_{1}^{\prime}\left(s_{4}\right)}^{v_{1}^{\prime}\left(s_{3}\right)} \frac{\mathrm{d} t}{g_{1}(|t|)} & =\int_{s_{4}}^{s_{3}} \frac{v_{1}^{\prime \prime}(s)}{g_{1}\left(\left|v_{1}^{\prime}(s)\right|\right)} \mathrm{d} s \\
& =\int_{s_{4}}^{s_{3}} \frac{-\hat{H}_{1}\left(s, v_{1}(s), v_{2}(s), v_{1}^{\prime}(s), v_{2}^{\prime}(s)\right)+\psi v_{1}(s)}{g_{1}\left(\left|v_{1}^{\prime}(s)\right|\right)} \mathrm{d} s \\
& =\int_{s_{4}}^{s_{3}} \frac{-h_{1}\left(s, v_{1}(s), v_{2}(s), v_{1}^{\prime}(s), v_{2}^{\prime}(s)\right)}{g_{1}\left(\left|v_{1}^{\prime}(s)\right|\right)} \mathrm{d} s
\end{aligned}
$$




$$
\begin{aligned}
& \leq \int_{s_{4}}^{s_{3}} \frac{\left|h_{1}\left(s, v_{1}(s), v_{2}(s), v_{1}^{\prime}(s), v_{2}^{\prime}(s)\right)\right|}{g_{1}\left(\left|v_{1}^{\prime}(s)\right|\right)} \mathrm{d} s \\
& \leq \int_{s_{4}}^{s_{3}} \frac{g_{1}\left(\left|v_{1}^{\prime}(s)\right|\right)}{g_{1}\left(\left|v_{1}^{\prime}(s)\right|\right)} \mathrm{d} s \\
& =\int_{s_{4}}^{s_{3}} \mathrm{~d} s \\
& =s_{3}-s_{4} \leq 1<\int_{p_{1}}^{G_{1}} \frac{\mathrm{d} t}{g_{1}(|t|)} .
\end{aligned}
$$

Hence $v_{1}^{\prime}\left(s_{3}\right)<G_{1}$ and, as $s_{3}$ is taken arbitrarily, $v_{1}^{\prime}\left(s_{3}\right)<G_{1}$ for values of $s$, where $v_{1}^{\prime}(s)>p_{1}$.

If $s_{2}>s_{3}$, the technique is analogous for $s_{4} \in\left[s_{3}, s_{2}\right]$. The same conclusion can be achieved if there are $s_{2}, s_{3} \in[0,1]$ such that

$$
v_{1}^{\prime}\left(s_{2}\right)>-p_{1} \quad \text { and } \quad v_{1}^{\prime}\left(s_{3}\right)<-p_{1} \text {. }
$$

Consequently, $\left\|v_{1}^{\prime}\right\| \leq G_{1}$, and on the same arguments, it can be easily proved that $\left\|v_{2}^{\prime}\right\| \leq$ $G_{2}$.

\section{Examples}

In this section, the applicability of the results developed in Sect. 3 is illustrated by taking examples. The first example is considered with nonlinear coupled BCs, and the assumptions of Theorem 1 are verified. In the second example, we consider the fully nonlinear coupled mass-spring model with coupled Dirichlet BCs, and the assumptions of Theorem 1 are verified.

Example 1 Consider the fully nonlinear coupled system

$$
\begin{aligned}
-v_{1}^{\prime \prime}(s)= & -v_{1}^{\prime}(s) v_{2}(s)+\arctan \left(v_{1}(s) v_{2}^{\prime}(s)\right)-v_{1}^{\prime}(s) \\
& -8 v_{1}(s)-4+s, \quad s \in[0,1], \\
-v_{2}^{\prime \prime}(s)= & -s^{2} \exp \left(-\left|v_{1}^{\prime}(s)\right|\right) v_{2}(s)+s^{2} v_{1}(s)\left(v_{2}^{\prime}(s)-2\right) \\
& -v_{2}^{\prime}(s)-4 v_{1}(s)-8, \quad s \in[0,1],
\end{aligned}
$$

subject to nonlinear coupled BCs

$$
\begin{aligned}
& \left(v_{1}^{2}(0) \sin \left(v_{2}(0)\right)-\sin \left(v_{2}(1)\right), v_{1}(1) \cos \left(v_{2}(1)\right)-2 \cos \left(v_{1}(0)\right)\right) \\
& \quad=(0,0) \\
& \left(v_{1}(0) \tan \left(v_{2}(0)\right)-\sin \left(v_{1}^{\prime}(1)\right) v_{2}^{\prime}(1),-v_{1}^{\prime}(1) \tan \left(v_{2}^{\prime}(1)\right)+v_{2}(0) v_{1}(1)\right) \\
& \quad=(0,0)
\end{aligned}
$$

This problem is a particular case of system (1), (2) with

$$
\begin{aligned}
& h_{1}\left(s, x_{0}, x_{1}, x_{2}, x_{3}\right)=-x_{2} x_{1}+\arctan \left(x_{0} x_{3}\right)-x_{2}-8 x_{1}-4+s \\
& h_{2}\left(s, x_{0}, x_{1}, x_{2}, x_{3}\right)=-s^{2} \exp \left(-\left|x_{2}\right|\right) x_{1}+s^{2} x_{0}\left(x_{3}-2\right)-x_{3}-4 x_{1}-8,
\end{aligned}
$$

continuous functions $s \in[0,1]$. 
The functions given by

$$
\left(\gamma_{1}(s), \gamma_{2}(s)\right)=(-2,-4) \quad \text { and } \quad\left(\xi_{1}(s), \xi_{2}(s)\right)=\left(s^{2}, s^{4}\right)
$$

are the lower and upper solutions of problem (50)-(51) satisfying (1), as

$$
\begin{aligned}
& \gamma_{1}^{\prime \prime}(s)=0<h_{1}(s,-2,-4,0,0)=12+s, \quad s \in[0,1], \\
& \gamma_{2}^{\prime \prime}(s)=0 \leq h_{2}(s,-2,-4,0,0)=8 s^{2}, \quad s \in[0,1]
\end{aligned}
$$

and

$$
\begin{aligned}
& \xi_{1}^{\prime \prime}(s)=-2>h_{1}\left(s, s^{2}, s^{4}, 2 s, 4 s^{3}\right)=-2 s^{5}+\arctan \left(4 s^{5}\right)-s-8 s^{2}-4, \quad s \in[0,1], \\
& \xi_{2}^{\prime \prime}(s)=-12 s^{2}>h_{2}\left(s, s^{2}, s^{4}, 2 s, 4 s^{3}\right)=s^{4}\left(4 s^{3}-2\right)-\frac{s^{6}}{\exp (2 s)}-4 s^{3}-4 s^{2}-8, \quad s \in[0,1] .
\end{aligned}
$$

Also, the functions $\gamma_{1}(s)=-2, \gamma_{2}(s)=-4, \xi_{1}(s)=s^{2}$, and $\xi_{2}(s)=s^{4}$ are coupled lower and upper solutions satisfying (35). Moreover, the continuous functions $h_{1}$ and $h_{2}$, as given in (52), satisfy the Nagumo condition relative to the intervals $\left[-2, s^{2}\right]$ and $\left[-4, s^{4}\right]$ for $s \in[0,1]$. For this, we first need to calculate $P_{1}$ and $P_{2}$ using (9) and (10) as

$$
\begin{aligned}
& P_{1}:=\max \left\{\xi_{1}(1)-\gamma_{1}(0), \xi_{1}(0)-\gamma_{1}(1)\right\}=3, \\
& P_{2}:=\max \left\{\xi_{2}(1)-\gamma_{2}(0), \xi_{2}(0)-\gamma_{2}(1)\right\}=5 .
\end{aligned}
$$

Also

$$
\left|h_{1}\left(s, x_{0}, x_{1}, x_{2}, x_{3}\right)\right| \leq 13+\frac{\pi}{2}+2\left|x_{2}\right|:=g_{1}\left(\left|x_{2}\right|\right)
$$

and

$$
\int_{P_{1}=3}^{G_{1}} \frac{\mathrm{d} s}{g_{1}(|s|)}=\int_{P_{1}=3}^{G_{1}} \frac{1}{13+\frac{\pi}{2}+2|s|} \mathrm{d} s>1
$$

for $G_{1} \geq 70$. Similarly,

$$
\left|h_{2}\left(s, x_{0}, x_{1}, x_{2}, x_{3}\right)\right| \leq 13+2\left|x_{3}-2\right|:=g_{2}\left(\left|x_{3}\right|\right)
$$

and

$$
\int_{P_{2}=5}^{G_{2}} \frac{\mathrm{d} s}{g_{2}(|s|)}=\int_{P_{2}=5}^{G_{2}} \frac{1}{13+2|s-2|} \mathrm{d} s>1
$$

for $G_{2} \geq 73$.

Then, by Theorem 1 , there exists at least one solution $\left(v_{1}(s), v_{2}(s)\right) \in C^{2}([0,1], \mathbb{R}) \times$ $C^{2}([0,1], \mathbb{R})$ of problem $(50)-(51)$ with

$$
\begin{aligned}
& -2 \leq v_{1}(s) \leq s^{2}, \quad-4 \leq v_{2}(s) \leq s^{4}, \quad \forall s \in[0,1] \\
& \left\|v_{1}^{\prime}\right\| \leq G_{1}, \quad \text { and } \quad\left\|v_{2}^{\prime}\right\| \leq G_{2} .
\end{aligned}
$$




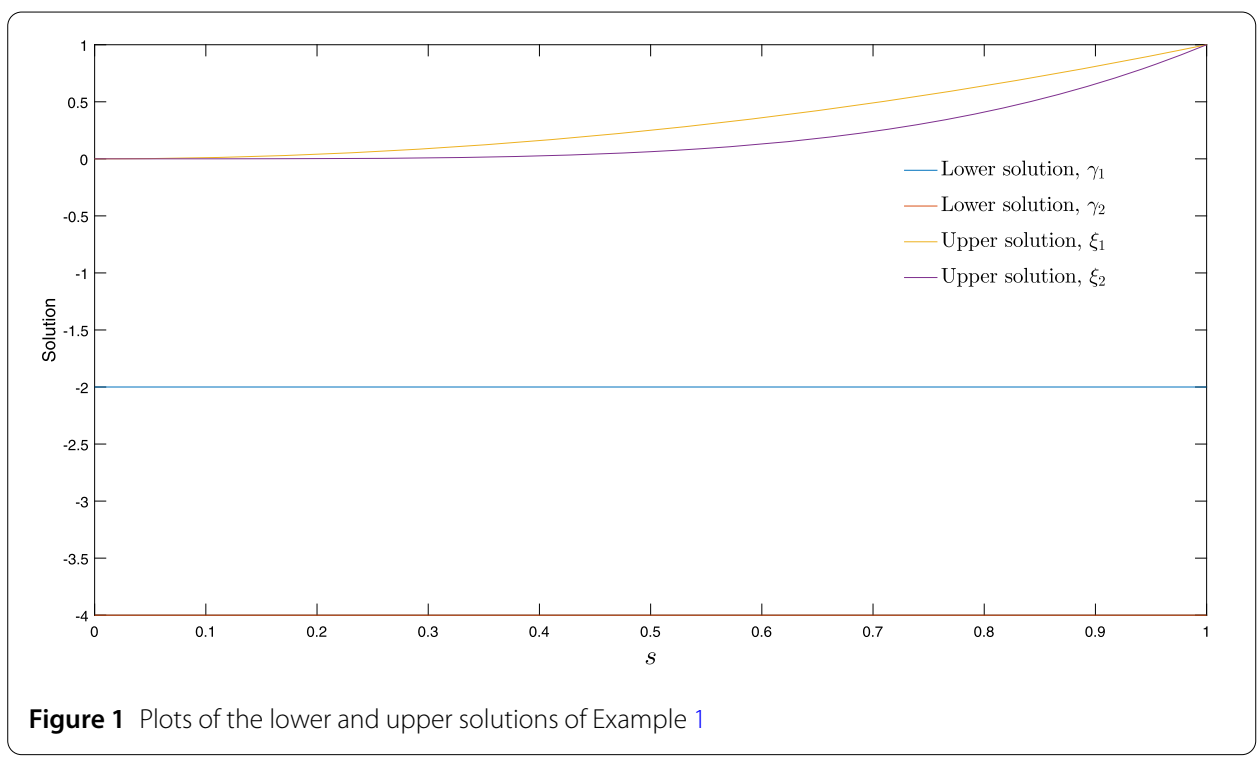

Figure 2 Full nonlinear coupled mass-spring model

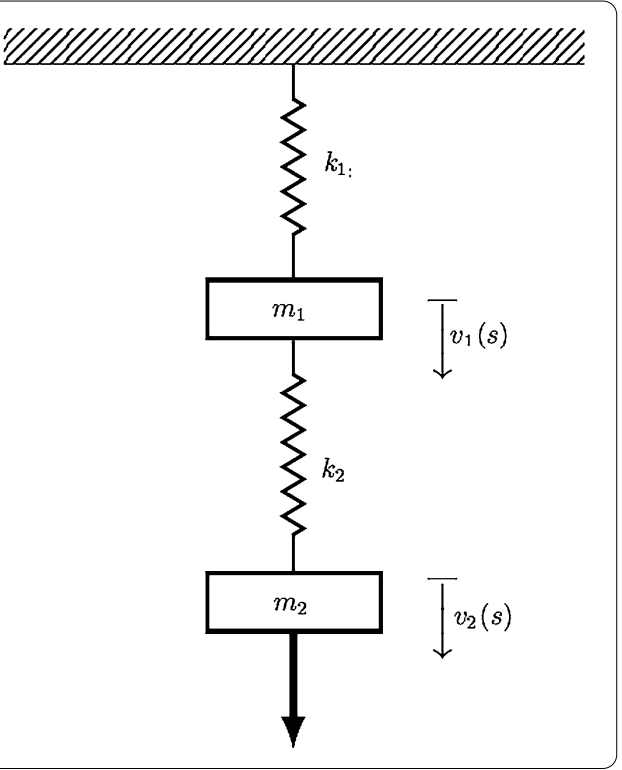

Example 2 Consider a coupled mass-spring model with two springs and two weights. The one end of the first spring having spring constant $k_{1}$ is attached to the ceiling, and a weight of mass $m_{1}$ is attached to its other end. To this weight, a second spring having spring constant $k_{2}$ is attached. A weight of mass $m_{2}$ is attached to the bottom end of the second spring. The entire coupled mass-spring model is described as illustrated in Fig. 2.

If the system is allowed to come to rest in equilibrium, then the displacement from the equilibrium position of the center of mass of each weight can be determined by considering them as a function of time $s$. Let the functions $v_{1}(s)$ and $v_{2}(s)$ denote these measurements. Consider $s \in[0,1]$, then $v_{1}(0)$ and $v_{2}(0)$ are the initial displacements of the mass $m_{1}$ and $m_{2}$, respectively. Similarly, $v_{1}(1)$ and $v_{2}(1)$ are the final displacements.

By considering the effects of viscous damping, restoring forces, and external forces of sinusoidal type, the coupled mass-spring model can be described by the second-order fully 
nonlinear ordinary coupled differential system [14]

$$
\begin{aligned}
v_{1}^{\prime \prime}(s)= & \frac{1}{m_{1}}\left(-\delta_{1} v_{1}^{\prime}(s)-k_{1} v_{1}(s)+\mu_{1}\left(v_{1}(s)\right)^{3}-k_{2}\left(v_{1}(s)-v_{2}(s)\right)+\mu_{2}\left(v_{1}(s)\right.\right. \\
& \left.\left.-v_{2}(s)\right)^{3}+F_{1} \cos \omega_{1} s\right) \\
v_{2}^{\prime \prime}(s)= & \frac{1}{m_{2}}\left(-\delta_{2} v_{2}^{\prime}(s)-k_{2}\left(v_{2}(s)-v_{1}(s)\right)+\mu_{2}\left(v_{2}(s)-v_{1}(s)\right)^{3}\right. \\
& \left.+F_{2} \cos \omega_{2} s\right)
\end{aligned}
$$

subject to nonlinear coupled boundary conditions of Dirichlet type

$$
\begin{aligned}
& \left(v_{1}^{2}(1)-\sin (s)-\cos \left(v_{2}^{\prime}(0)\right), v_{1}(0)\right)=(0,0), \\
& \left(v_{2}^{2}(1)-\cos (s)-\cos \left(v_{1}(0)\right), v_{2}(0)\right)=(0,0) .
\end{aligned}
$$

The boundary conditions (55) can also be expressed as

$$
\begin{aligned}
& v_{1}(0)=0, \\
& v_{1}(1)=\sqrt{\sin (s)+\cos \left(v_{2}^{\prime}(0)\right)}, \\
& v_{2}(0)=0 \\
& v_{2}(1)=\sqrt{\cos (s)+\cos \left(v_{1}(0)\right)} .
\end{aligned}
$$

The boundary conditions (56) have physical meanings, at $s=0$ the initial displacements of the mass $m_{1}$ and $m_{2}$ are $v_{1}(0)$ and $v_{2}(0)$, respectively, and at $s=1$ the final displacement for the first mass is $v_{1}(1)$, which is the sum of sinusoidal sine function in this period of time, with the initial velocity of the second mass as an argument of the cosine function. Similarly, $v_{2}(1)$ is the final displacement for the second mass, which is the sum of sinusoidal cosine function in this period of time, with the initial displacement of the first mass as an argument of the cosine function.

Model (54)-(56) is a particular case of system (1), (2) with

$$
\begin{aligned}
h_{1}\left(s, x_{0}, x_{1}, x_{2}, x_{3}\right)= & \frac{1}{m_{1}}\left(-\delta_{1} x_{2}-k_{1} x_{0}+\mu_{1}\left(x_{0}\right)^{3}-k_{2}\left(x_{0}-x_{1}\right)\right. \\
& \left.+\mu_{2}\left(x_{0}-x_{1}\right)^{3}+F_{1} \cos \omega_{1} s\right) \\
h_{2}\left(s, x_{0}, x_{1}, x_{2}, x_{3}\right)= & \frac{1}{m_{2}}\left(-\delta_{2} x_{3}-k_{2}\left(x_{1}-x_{0}\right)+\mu_{2}\left(x_{1}-x_{0}\right)^{3}\right. \\
& \left.+F_{2} \cos \omega_{2} s\right),
\end{aligned}
$$

continuous functions for $s \in[0,1]$. Moreover, $\delta_{1}, \delta_{2}$ are the damping coefficients, $\mu_{1}, \mu_{2}$ are the coefficients of each nonlinear term of the coupled system, $k_{2}\left(v_{1}(s)-v_{2}(s)\right)+\mu_{2}\left(v_{1}(s)-\right.$ $\left.v_{2}(s)\right)^{3}, k_{2}\left(v_{2}(s)-v_{1}(s)\right)+\mu_{2}\left(v_{2}(s)-v_{1}(s)\right)^{3}$ are the restoring forces, and $F_{1} \cos \omega_{1} s, F_{2} \cos \omega_{2} s$ are the external sinusoidal forces with forcing amplitudes $F_{1}, F_{2}$ and forcing frequencies $\omega_{1}, \omega_{2}$. 
For each nonnegative $m_{1}, m_{2}, F_{1}, F_{2}, \omega_{1}, \omega_{2}, \delta_{1}, \delta_{2}$, and any real $\mu_{1}, \mu_{2}, k_{1}, k_{2}$, such that for

$$
\begin{aligned}
& F_{1} \leq \delta_{1}, \\
& F_{2} \leq \delta_{2}, \\
& \mu_{1} \leq 0, \mu_{2} \leq 0,
\end{aligned}
$$

the functions given by

$$
\left(\gamma_{1}(s), \gamma_{2}(s)\right)=(-s,-4 s) \text { and }\left(\xi_{1}(s), \xi_{2}(s)\right)=\left(s^{4}, s^{4}\right)
$$

are the lower and upper solutions of problem (54)-(56) satisfying (1), as

$$
\begin{aligned}
\gamma_{1}^{\prime \prime}(s) & =0 \leq \frac{1}{m_{1}}\left(\delta_{1}-F_{1}\right) \\
& \leq \frac{1}{m_{1}}\left(\delta_{1}+k_{1} s-\mu_{1} s^{3}+3 k_{2} s-27 \mu_{2} s^{3}+F_{1} \cos \omega_{1} s\right), \quad \forall s \in[0,1] \\
& =h_{1}(s,-s,-4 s,-1,-4), \\
\gamma_{2}^{\prime \prime}(s) & =0 \leq \frac{1}{m_{2}}\left(\delta_{2}-F_{2}\right) \\
& \leq \frac{1}{m_{2}}\left(4 \delta_{2}+3 k_{2} s-27 \mu_{2} s^{3}+F_{2} \cos \omega_{2} s\right), \quad \forall s \in[0,1] \\
& =h_{2}(s,-s,-4 s,-1,-4)
\end{aligned}
$$

and

$$
\begin{aligned}
\xi_{1}^{\prime \prime}(s) & =12 s^{2} \geq \frac{1}{m_{1}}\left(-\delta_{1}+F_{1}\right) \\
& \geq \frac{1}{m_{1}}\left(-4 \delta_{1} s^{3}-k_{1} s^{4}+\mu_{1} s^{12}+F_{1} \cos \omega_{1} s\right), \quad \forall s \in[0,1] \\
& =h_{1}\left(s, s^{4}, s^{4}, 4 s^{3}, 4 s^{3}\right), \\
\xi_{2}^{\prime \prime}(s) & =12 s^{2} \geq \frac{1}{m_{2}}\left(-\delta_{2}+F_{2}\right) \\
& \geq \frac{1}{m_{2}}\left(-4 \delta_{2} s^{3}+F_{2} \cos \omega_{2} s\right), \quad \forall s \in[0,1] \\
& =h_{2}\left(s, s^{4}, s^{4}, 4 s^{3}, 4 s^{3}\right) .
\end{aligned}
$$

Also, the functions $\gamma_{1}(s)=-s, \gamma_{2}(s)=-4 s, \xi_{1}(s)=s^{4}$, and $\xi_{2}(s)=s^{4}$ are coupled lower and upper solutions satisfying (35). Moreover, the continuous functions $h_{1}$ and $h_{2}$, as given in (57), satisfy the Nagumo condition relative to the intervals $\left[-s, s^{4}\right]$ and $\left[-4 s, s^{4}\right]$ for $s \in[0,1]$. For this, we first need to calculate $P_{1}$ and $P_{2}$ using (9) and (10) as follows:

$$
\begin{aligned}
& P_{1}:=\max \left\{\xi_{1}(1)-\gamma_{1}(0), \xi_{1}(0)-\gamma_{1}(1)\right\}=1, \\
& P_{2}:=\max \left\{\xi_{2}(1)-\gamma_{2}(0), \xi_{2}(0)-\gamma_{2}(1)\right\}=4 .
\end{aligned}
$$




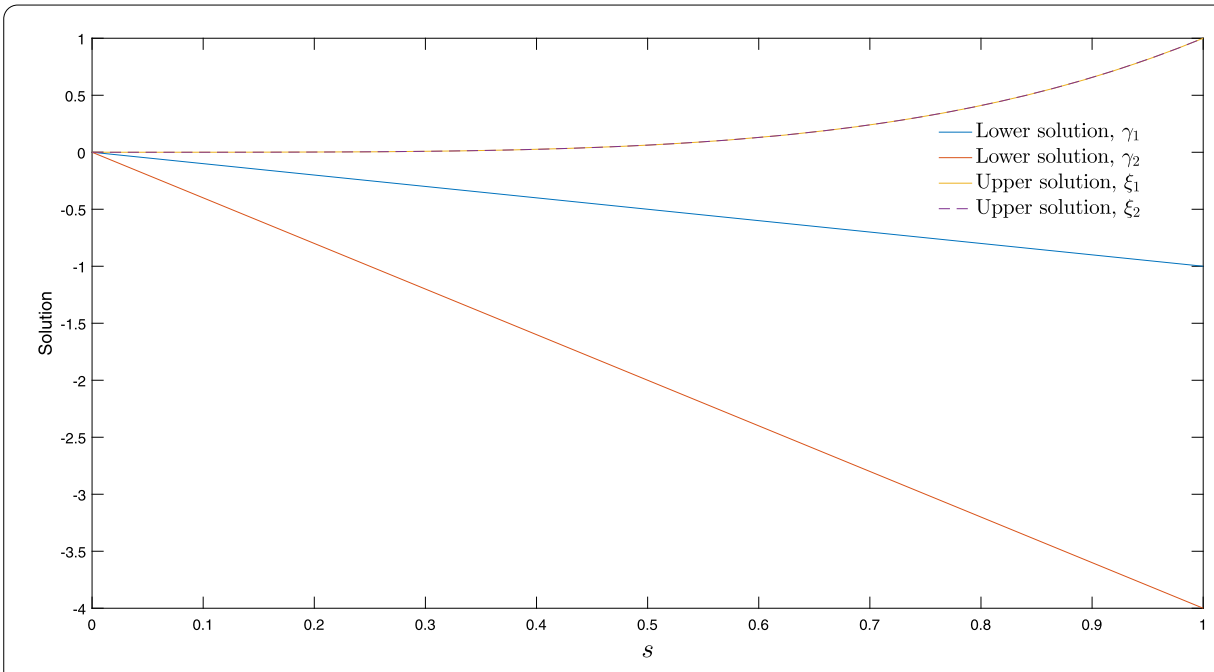

Figure 3 Plots of the lower and upper solutions of Example 2

Also

$$
\left|h_{1}\left(s, x_{0}, x_{1}, x_{2}, x_{3}\right)\right| \leq \delta_{1}\left|x_{2}\right|+F_{1}+8\left|\mu_{2}\right|+\left|\mu_{1}\right|+2 k_{2}:=g_{1}\left(\left|x_{2}\right|\right),
$$

and for large $G_{1}$, we have

$$
\int_{P_{1}=1}^{G_{1}} \frac{\mathrm{d} s}{g_{1}(|s|)}=\int_{P_{1}=1}^{G_{1}} \frac{m_{1}}{\delta_{1}|s|+F_{1}+8\left|\mu_{2}\right|+\left|\mu_{1}\right|+2 k_{2}} \mathrm{~d} s>1 .
$$

Similarly,

$$
\left|h_{2}\left(s, x_{0}, x_{1}, x_{2}, x_{3}\right)\right| \leq \delta_{2}\left|x_{3}\right|+F_{2}+8\left|\mu_{2}\right|+2 k_{2}:=g_{2}\left(\left|x_{3}\right|\right),
$$

and for large $G_{2}$, we have

$$
\int_{P_{2}=4}^{G_{2}} \frac{\mathrm{d} s}{g_{2}(|s|)}=\int_{P_{2}=4}^{G_{2}} \frac{m_{2}}{\delta_{2}|s|+F_{2}+8\left|\mu_{2}\right|+2 k_{2}} \mathrm{~d} s>1 .
$$

Then, by Theorem 1 , there exists at least one solution $\left(v_{1}(s), v_{2}(s)\right) \in C^{2}([0,1], \mathbb{R}) \times$ $C^{2}([0,1], \mathbb{R})$ of the coupled mass-spring model (54)-(56) with

$$
\begin{aligned}
& -s \leq v_{1}(s) \leq s^{4}, \quad-4 s \leq v_{2}(s) \leq s^{4}, \quad \forall s \in[0,1] \\
& \left\|v_{1}^{\prime}\right\| \leq G_{1}, \quad \text { and } \quad\left\|v_{2}^{\prime}\right\| \leq G_{2} .
\end{aligned}
$$

\section{Conclusion}

In this article, we presented the generalized results based on the LUSs approach to study the existence of solutions of second-order fully nonlinear coupled systems with generalized nonlinear coupled boundary conditions. Our presented results have ability to study the existence of solutions of certain BVPs in a unified way which has been previously studied on a case-by-case basis. For instance, Dirichlet and Neumann are the special cases. We 
demonstrated the applicability of the developed theoretical results by considering some examples. We also extended the results presented in [1-4].

\author{
Acknowledgements \\ This research was funded by the Deanship of Scientific Research at Princess Nourah bint Abdulrahman University, Saudi \\ Arabia through the Fast-track Research Funding Program.
}

\title{
Funding
}

Not applicable.

Availability of data and materials

Not applicable.

Competing interests

The authors declare that they have no competing interests.

\section{Authors' contributions}

All authors contributed equally to this article. All authors read and approved the final manuscript.

\section{Author details}

${ }^{1}$ Nonlinear Analysis Group (NAG), Mathematics Department, Virtual University of Pakistan, 54-Lawrence Road, Lahore, Pakistan. ${ }^{2}$ Department of Mathematics and General Sciences, Prince Sultan University, Riyadh, Saudi Arabia. ${ }^{3}$ Department of Medical Research, China Medical University, 40402, Taichung, Taiwan. ${ }^{4}$ Department of Computer Science and Information Engineering, Asia University, Taichung, Taiwan. ${ }^{5}$ Department of Mathematical Sciences, Faculty of Sciences, Princess Nourah Bint Abdulrahman University, P.O. Box 84428, 11671, Riyadh, Saudi Arabia. ${ }^{6}$ Department of Mathematics, Faculty of Sciences, Van Yuzuncu Yil University, Van, Turkey.

\section{Publisher's Note}

Springer Nature remains neutral with regard to jurisdictional claims in published maps and institutional affiliations.

Received: 17 January 2021 Accepted: 25 July 2021 Published online: 06 August 2021

\section{References}

1. Franco, D., Nieto, J.J., O'Regan, D.: Upper and lower solutions for first order problems with nonlinear boundary conditions. Extr. Math. 18(2), 153-160 (2003)

2. Franco, D., Nieto, J.J., O'Regan, D.: Existence of solutions for first order ordinary differential equations with nonlinear boundary conditions. Appl. Math. Comput. 153, 793-802 (2004)

3. Franco, D., O'Regan, D.: A new upper and lower solutions approach for second order probems with nonlinear boundary conditions. Arch. Inequal. Appl. 1, 423-430 (2003)

4. Asif, N.A., Talib, I., Tunc, C.: Existence of solution for first-order coupled system with nonlinear coupled boundary conditions. Bound. Value Probl. 2015, 134 (2015)

5. Lian, W.C., Wong, F.H., Yeh, C.C.: On the existence of positive solutions of nonlinear second order differential equations. Proc. Am. Math. Soc. 124(4), 1117-1126 (1996)

6. Tisdell, C.C.: Existence of solutions to second-order boundary value problems without growth restrictions. Electron. J. Qual. Theory Differ. Equ. 2016, 92 (2016)

7. Infante, G., Pietramala, P.: Nonzero radial solutions for a class of elliptic systems with nonlocal BCs on annular domains. NoDEA Nonlinear Differ. Equ. Appl. 22, 979-1003 (2015)

8. Figueiredo, D.G.D., Ubilla, P.: Superlinear systems of second-order ODE's. Nonlinear Anal., Theory Methods Appl. 68(6), 1765-1773 (2008)

9. Cianciaruso, F., Infante, G., Pietramala, P.: Multiple positive radial solutions for Neumann elliptic systems with gradient dependence. Math. Methods Appl. Sci. 41(16), 6358-6367 (2018)

10. Abbas, S., Benchohra, M., Henderson, J., Lazreg, J.E.: Weak solutions for a coupled system of partial Pettis Hadamard fractional integral equations. Adv. Theory Nonlinear Anal. Appl. 1(2), 136-146 (2017)

11. Infante, G., Webb, J.R.L.: Loss of positivity in a nonlinear scalar heat equation. NoDEA Nonlinear Differ. Equ. Appl. 13, 249-261 (2006)

12. Guidotti, P., Merino, S.: Gradual loss of positivity and hidden invariant cones in a scalar heat equation. Differ. Integral Equ. 13, 1551-1568 (2000)

13. Webb, J.R.L.: Existence of positive solutions for a thermostat model. Nonlinear Anal., Real World Appl. 13, 923-938 (2012)

14. Temple, H.F.: Coupled spring equations. Int. J. Math. Educ. Sci. Technol. 34(1), 65-79 (2003)

15. Shi, Y.D., Zhou, Q.D., Li, Y.: A note on a two-point boundary value problem arising from a liquid metal flow. SIAM J. Math. Anal. 28(5), 1086-1093 (1997)

16. Horgan, C.O., Saccomandi, G., Sgura, I.: A two-point boundary-value problem for the axial shear of hardening isotropic incompressible nonlinearly elastic materials. SIAM J. Appl. Math. 62(5), 1712-1727 (2002)

17. Amster, P., Rogers, C.: On boundary value problems in three-ion electrodiffusion. J. Math. Anal. Appl. 333(1), 42-51 (2007)

18. Amara, J.B.: Sturm theory for the equation of vibrating beam. J. Math. Anal. Appl. 349(1), 1-9 (2009)

19. Amann, H.: Parabolic evolution and nonlinear boundary conditions. J. Differ. Equ. 72(1), $201-269$ (1988)

20. Aris, R.: Introduction to the Analysis of Chemical Reactors. Prentice Hall, Englewood Cliffs (1965) 
21. Aronson, D.G.: A comparison method for stability analysis of nonlinear parabolic problems. SIAM Rev. 20(2), 245-264 (1978)

22. Leung, A.: A semilinear reaction-diffusion prey-predator system with nonlinear coupled boundary conditions: equilibrium and stability. Indiana Univ. Math. J. 31, 223-241 (1982)

23. Mehmeti, F.A., Nicaise, S.: Nonlinear interaction problems. Nonlinear Anal. 20(1), $27-61$ (1993)

24. Wang, A., Sun, J., Zettle, A.: The classification of self-adjoint boundary conditions: separated, coupled. J. Funct. Anal. 255, 1554-1573 (2008)

25. Ascher, U.M., Mattheij, R.M.M., Russell, R.D.: Numerical Solution of Boundary Value Problems for Ordinary Differential Equations. Classics in Applied Mathematics. SIAM, Philadelphia (1995). Corrected Reprint of the 1988 Original

26. Agarwal, R.P.: Boundary Value Problems for Higher Order Differential Equations. World Scientific, Teaneck (1986)

27. Jarad, F., Abdeljawad, T:: A modified Laplace transform for certain generalized fractional operators. Results Nonlinear Anal. 1(2), 88-98 (2018)

28. Redhwan, S.S., Shaikh, S.L., Abdo, M.S.: Some properties of Sadik transform and its applications of fractional-order dynamical systems in control theory. Ad. Theory Nonlinear Anal. Appl. 4(1), 51-66 (2020)

29. Abdeljawad, T., Agarwal, R.P., Karapinar, E., Kumari, P.S.: Solutions of the nonlinear integral equation and fractional differential equation using the technique of a fixed point with a numerical experiment in extended $b$-metric space. Symmetry 11(5), 686 (2019)

30. Karapinar, E., Fulga, A., Rashid, M., Shahid, L., Aydi, H.: Large contractions on quasi-metric spaces with an application to nonlinear fractional differential equations. Mathematics 7(5), 444 (2019)

31. Alqahtani, B., Aydil, H., Karapinar, E., Rakočević, V.: A solution for Volterra fractional integral equations by hybrid contractions. Mathematics 7(8), 694 (2019)

32. Afshari, H., Kalantari, S., Karapinar, E.: Solution of fractional differential equations via coupled fixed points. Electron J. Differ. Equ. 2015, 286 (2015)

33. Adigüzel, R.S., Aksoy, U., Karapinar, E., Erhan, I.M.: On the solution of a boundary value problem associated with a fractional differential equation. Math. Methods Appl. Sci. (2020). https://doi.org/10.1002/mma.6652

34. Talib, I., Asif, N.A., Tunc, C.: Coupled lower and upper solution approach for the existence of solutions of nonlinear coupled system with nonlinear coupled boundary conditions. Proyecciones 35(1), 99-117 (2016)

35. Minhós, F., Fialho, J., de Sousa, R.: Periodic $n$ dimensional first order coupled systems and periodic schizophrenia phenomena. J. Math. Anal. Appl. 492(2), 124482 (2020)

36. Akô, K.: Subfunctions for ordinary differential equations. Funkc. Ekvacioj 12, 239-241 (1969)

37. Knobloch, H.W.: Comparison theorems for nonlinear second order differential equations. J. Differ. Equ. 1, 1-26 (1965)

38. Schmitt, K.: Boundary value problems for nonlinear second order differential equations. Monatshefte Math. 176, 347-354 (1968)

39. Kelevedjiev, P.: Existence of solutions for two-point boundary value problems. Nonlinear Anal. 22, 217-224 (1994)

40. Granas, A., Guenther, R., Lee, J.: Nonlinear boundary value problems for ordinary differential equations. Diss. Math. 244, 1-128 (1985)

41. Korman, P.: Remarks on Nagumo condition. Port. Math. 55, 1-9 (1998)

42. Nagumo, M.: Uber die Differentialgleichung $y^{\prime \prime}=f\left(x, y, y^{\prime}\right)$. Proc. Phys. Math. Soc. Jpn. 9, 861-866 (1937) (in German)

43. Nagumo, M.: Collected Papers. Edited by Masaya Yamaguti, Louis Nirenberg, Sigeru Mizohata and Yasutaka Sibuya. With Appendices by Yamaguti, Mizohata, Sibuya and Michihiro Nagase. Springer, Tokyo (1993)

44. Bernstein, S.R.: Sur les équations du calcul des variations. Ann. Sci. Éc. Norm. Supér. 29, 431-485 (1912) (in French)

\section{Submit your manuscript to a SpringerOpen ${ }^{\circ}$ journal and benefit from:}

- Convenient online submission

- Rigorous peer review

- Open access: articles freely available online

- High visibility within the field

- Retaining the copyright to your article

Submit your next manuscript at $\gg$ springeropen.com 